\title{
Modelo do usuário sensível ao contexto cultural em um sistema e-learning adaptativo
}

\section{Culturally-aware user model in an adaptive e-learning system}

\begin{abstract}
Resumo: Este artigo apresenta uma nova abordagem para a modelagem do aluno em sistemas de e-learning visando melhorar as capacidades de personalização e levando em consideração varias perspectivas complementares de contexto, tais como os contextos pessoal, educacional, tecnológico e especialmente o contexto cultural. As principais caracteristicas da nossa abordagem são descritas e ilustradas, em particular detalhando (i) a adoção de ontologias para representação de modelos de contexto e (ii) como o contexto cultural poder ser modelado como uma extensão do tradicional modelo do aluno.

Palavras-chave: Contexto cultural. Adaptação. Sensibilidade ao contexto. Modelo do usuário estendido, e-learning.

Abstract: This paper presents a new approach to student modeling in e-leaning to improve personalization capabilities and taking into account various complementary perspectives of context, such as personal, educational, technological, and erecilly are described and illustrated, particularly detailing (i) the (ii) how cultural context can be modeled as an extension of traditional student model. Keymords: Cultural cont Adaptation. Context-aware. Extended user model. E-learning.
\end{abstract} GASPARINI, Isabela; KEMCZINSKI, Avanilde; PIMENTA, Marcelo, Soares; PALAZZO M. de Oliveira, Jose. Modelo do usuário sensivel ao contexto cultural em um sistema e-learning adaptativo. Informatica na Educaçao: teoria \& 2011.
Isabela Gasparini

Universidade do Estado de Santa Catarina Universidade Federal do Rio Grande do Sul

\section{Avanilde Kemczinski}

Universidade do Estado de Santa Catarina

\section{Marcelo Soares Pimenta}

Universidade Federal do Rio Grande do Sul

\section{José Palazzo Moreira de Oliveira}

Universidade Federal do Rio Grande do Sul

\section{Introdução}

$\mathrm{U}$ m sistema de e-learning é normalmente utilizado por uma variedade de alunos, com diferentes habilidades, experiências, preferências e estilos de aprendizagem. Assim, uma das características mais desejáveis dos sistemas educacionais é ser adaptativo ou personalizado (Brusilosvky e Peylo, 2003). Um sistema e-learning adaptativo ajusta o conteúdo, a apresentação e a navegação de acordo com o modelo do usuário/aluno. Personalização (ou adaptabilidade) é o processo de adaptação de uma aplicação computacional às necessidades dos usuários, e se aproveita do conhecimento adquirido sobre eles. Na verdade, o uso de técnicas de personalização objetivam melhorar a usabilidade e a interação dos sistemas e-learning, uma vez que o sistema 
adapta a interface, o conteúdo, a apresentação e a navegação, considerando o perfil do usuário (normalmente chamado de modelo do aluno) e cada usuário tem a percepção de que o sistema foi projetado especialmente para ele. Os sistemas adaptativos trazem um novo panorama, modificando o conceito tradicional ainda existente na área da computação - "one size fits all", em que vários usuários, com diferentes características, utilizam a mesma interface padronizada.

Geralmente, as características mais amplamente utilizadas no modelo do aluno são: o interesse do aluno, seu conhecimento adquirido sobre o domínio, suas características individuais, suas experiências prévias, seus objetivos e suas tarefas dentro do ambiente e-learning (Brusilovsky e Millan, 2007). Entretanto, os sistemas elearning podem ser dinamicamente ajustados não só de acordo com o modelo do aluno, mas também em função de uma noção mais rica do contexto do usuário. Um sistema e-learning contextualizado proporciona ao aluno o material exato que ele necessita e adequado ao seu nível de conhecimento e que faz sentido em uma situação especial de aprendizagem, chamada de cenário em nosso trabalho.

Um dos objetivos da nossa pesquisa é investigar abordagens para colocar o perfil dos usuários e o conhecimento contextual em prática no processo de desenvolvimento de sistemas e-learning - particularmente em um sistema adaptativo de aprendizagem chamado AdaptWeb ${ }^{\circledR}$.

O objetivo deste artigo é apresentar nossa abordagem para a modelagem do contexto do usuário, especialmente o contexto cultural, para tornar o ambiente com maior capacidade de adaptação.

Este artigo está estruturado como segue. A seção 2 apresenta a definição sobre cultura, bem como as dimensões propostas por Hofstede (2001) para a diferenciação da cultura nacional. A seção 3 discute sobre os aspectos culturais que podem ser utilizados no design da interação. A seção 4 apresenta os trabalhos relacionados, focando na temática da cultura nas áreas de web semântica e educação. A seção 5 detalha o ambiente AdaptWeb $^{\circledR}$, explorando seu funcionamento de adaptação baseado no modelo do aluno e sua estrutura de organização. A seção 6 explica nossa abordagem para modelagem contextual do aluno, estendendo o modelo tradicional do usuário. A seção 7 apresenta dois cenários de aplicação apresentando o melhoramento das capacidades de personalização disponibilizadas pela nossa abordagem. Finalmente na seção 8 é apresentada a conclusão deste trabalho, e em seguida nossos agradecimentos e as referências.

\section{Cultura}

No sentido mais geral, o termo "cultura" é usado por pesquisadores de campo social para se referir a um conjunto de parâmetros que podem ser usados para diferenciar um grupo social de forma significativa, tais como nações, empresas e grupos (AbouZeid, 2005). Uma definição aceita na literatura sobre a cultura é "a programação coletiva da mente que distingue membros de um grupo ou categoria de pessoas" (HOFSTEDE, 2005).

Geert Hofstede conduziu pesquisa detalhada com centenas de funcionários da IBM em 53 países, que, mais tarde foi expandida para 74 países. Ele foi capaz de determinar padrões de semelhanças e diferenças entre as respostas das entrevistas e desenvolveu um modelo que diferencia cinco dimensões culturais nacionais (Hofstede, 2005; Hofstede, 2001), e de fato seu trabalho parece ser a abordagem mais comum para a cultura na área de Interação Humano-Computador IHC (Clemmensen et al., 2009).

Estas dimensões são apresentadas a seguir. PDI (power of distance) - é o grau de igualdade (ou desigualdade) entre as pessoas de uma nação. Um alto valor para o PDI indica sociedades onde o crescimento da desigualdade de poder e riqueza é permitida. É o grau em que membros menos poderosos das organizações e instituições aceitam e esperam que o poder seja distribuído de forma desigual.

IDV (individualism) é o grau em que a sociedade reforça a realização individual ou coletiva e sobre as relações interpessoais. Um alto valor no índice de IDV indica uma 
sociedade mais individualista e os direitos individuais são fundamentais dentro da sociedade. Já um baixo índice IDV indica uma sociedade coletiva.

MAS (masculinity), é o grau que a sociedade reforça ou não o modelo tradicional do papel masculino de trabalho, da realização, controle e poder. Um alto valor neste índice indica países que possuem um alto grau de diferenciação pelo gênero.

UAI (uncertainty avoidance index) corresponde ao nível de tolerância sobre a incerteza e a ambigüidade que a sociedade possui para situações não-estruturadas. Um alto valor para este índice indica que o país possui uma baixa tolerância à incerteza.

LTO (long-term orientation index) é o grau que a sociedade adota ou não valores tracionais, pensando no futuro. Um alto valor LTO indica que o país prescreve os valores de compromissos de longo prazo e de respeito pela tradição, e valores baixos para o índice LTO indicam que o país não reforça este conceito. Para essas culturas, a mudança pode ocorrer mais rapidamente e o respeito a tradições e compromisso não são obstáculos para mudanças.

Estes cinco índices propostos por Hofstede (2001) incluem valores, estruturas cognitivas e comportamentos em diferentes níveis, tais como no nível individual, no nível das estruturas da organização, e sobre os artefatos e os atributos para o desenvolvimento social ou nacional (Zaharias, 2008).

\section{Aspectos Culturais e Interação Humano-Computador (IHC)}

$\mathrm{Na}$ perspectiva da organização do conhecimento, cultura, pela sua natureza, é um fenômeno em multicamadas, que pode ser manifestado em diversos níveis, como o nível da sociedade, o nível nacional, corporativo e ocupacional/profissional, que influenciam o comportamento social dos envolvidos em cada etapa do processo de transferência de conhecimento (Hofstede, 2005; Abou-Zeid, 2005). Além disso, para cada camada, há diferentes contextos culturais. O nível da sociedade lida com a sociedade em um nível epistemológico, por exemplo, as diferenças de percepção, interpretação e raciocínio sobre um fenômeno. Os outros três níveis podem ser descritos pelo conjunto de valores que distinguem uma organização de outra em um determinado nível de análise. O nível nacional inclui valores tanto abstrativos versus associativos proposto por Hofstede. No nível corporativo, alguns exemplos são a avaliação da disposição das informações, a disponibilidade para compartilhar conhecimentos com os outros e a vontade de aprender conjuntamente. O nível operacional/ocupacional pode ser representado por valores como estilos de gestão e delegação de responsabilidades.

Aspectos culturais são as preferências e modos de comportamento determinado pela cultura de uma pessoa. O contexto cultural, na área educacional, inclui a formação cultural do aluno e pode ter um grande impacto sobre sua capacidade e eficiência para aprender um determinado conjunto de conteúdos (Chandramouli et al., 2008). Um perfil cultural do aluno não pode ser definido como uma especificação fixa ou prescrita. A especificação deve ser ampliada e melhorada dinamicamente com base no contexto do usuário. Conforme descrito por Reinecke e Bernstein (2007), pesquisas realizadas sobre a usabilidade de interfaces e sites web culturalmente adaptados mostraram enormes melhorias na eficiência de trabalho.

O projeto da interface do usuário pode ser uma questão de preferência, que varia de pessoa para pessoa, contudo, existem preferências comuns que estão profundamente enraizadas na cultura (Dormann e Chisalita, 2002), e a investigação neste sentido mostra que pessoas consideradas pertencentes a um mesmo grupo cultural também percebem e processam informações de forma similar (Reinecke et al., 2010).

$\mathrm{Na}$ área de IHC, existem duas categorias principais que focam na adaptação (localização e globalização) baseada nos aspectos culturais: (i) a apresentação da informação (e.g. horário, data e formato de cor) e linguagem (e.g. fonte, direção de escrita, etc.), e; (ii) projeto do diálogo (e.g. estrutura do menu e complexidade, layout, posições) e design da interação (e.g. 
conceito da navegação, caminho da interação, velocidade da interação, estrutura do sistema, etc.) (Heimgärtner, 2007). Apesar de alguns trabalhos na área de IHC se concentrarem nos aspectos culturais, a pesquisa sobre os aspectos de IHC dependentes da cultura ainda é embrionária (Zaharias, 2008).

Um levantamento sobre os diversos trabalhos da área de IHC que tratam da adaptação cultural e indicam como o design da interação pode ser adaptado aos índices das dimensões de Hofstede é discutido em Gasparini, Pimenta e Palazzo (2011).

Além dos aspectos culturais para a adaptação do design da interação, Vatrapu e Suthers (2007) fizeram uma análise comparativa entre as dimensões culturais de Hofstede e o comportamento na aprendizagem em sala de aula. A tabela 1 apresenta a análise de Vatrapu e Suthers (2007).

Tabela 1 - Aspectos de aprendizagem versus dimensões culturais de Hofstede

\begin{tabular}{|c|c|c|}
\hline $\begin{array}{l}\text { Dimen } \\
\text { são }\end{array}$ & Valores baixos & Valores altos \\
\hline PDI & $\begin{array}{l}\text { Educação centrada } \\
\text { no estudante. } \\
\text { Os professores } \\
\text { esperam que os } \\
\text { alunos iniciem } \\
\text { comunicação. } \\
\text { Os alunos podem } \\
\text { espontaneamente se } \\
\text { manifestar nas } \\
\text { aulas. } \\
\text { É permitido aos } \\
\text { alunos contradizer } \\
\text { ou criticar o } \\
\text { professor. } \\
\text { A eficiência no } \\
\text { aprendizado é } \\
\text { relacionada com a } \\
\text { comunicação em } \\
\text { sala em "duas vias". } \\
\text { Fora da classe, os } \\
\text { professores são } \\
\text { tratados como } \\
\text { "iguais". }\end{array}$ & $\begin{array}{l}\text { Educação centrada no } \\
\text { professor. } \\
\text { Alunos esperam que } \\
\text { os professores } \\
\text { iniciem a } \\
\text { comunicação. } \\
\text { Os alunos geralmente } \\
\text { se manifestam nas } \\
\text { aulas quando o } \\
\text { professor solicita. } \\
\text { Professores não são } \\
\text { contraditos e nem } \\
\text { criticados } \\
\text { publicamente. } \\
\text { A eficiência na } \\
\text { aprendizagem é } \\
\text { relacionada com a } \\
\text { excelência do } \\
\text { professor. } \\
\text { O respeito pelo } \\
\text { professor também é } \\
\text { mostrado fora da sala } \\
\text { de aula. }\end{array}$ \\
\hline IDV & $\begin{array}{l}\text { Para sociedades } \\
\text { mais coletivas, os } \\
\text { alunos esperam } \\
\text { aprender como se } \\
\text { faz algo. } \\
\text { Indivíduos se } \\
\text { manifestarão nas } \\
\text { classes somente se } \\
\text { o professor os } \\
\text { chamar } \\
\text { pessoalmente. } \\
\text { Classes grandes se } \\
\text { dividem socialmente } \\
\text { em grupos menores, } \\
\text { baseando-se em } \\
\text { critérios } \\
\text { particulares. }\end{array}$ & $\begin{array}{l}\text { Para sociedades mais } \\
\text { individualistas, os } \\
\text { alunos esperam } \\
\text { aprender como } \\
\text { aprender. } \\
\text { Indivíduos se } \\
\text { manifestarão nas } \\
\text { classes se o professor } \\
\text { fizer uma pergunta a } \\
\text { classe como um todo. } \\
\text { A subdivisão varia de } \\
\text { uma situação a outra } \\
\text { (e.g. uma tarefa } \\
\text { particular). }\end{array}$ \\
\hline
\end{tabular}

\begin{tabular}{|c|c|c|}
\hline MAS & $\begin{array}{l}\text { Em sociedades mais } \\
\text { femininas, os } \\
\text { professores evitam } \\
\text { elogiar abertamente } \\
\text { os estudantes. } \\
\text { Os professores usam } \\
\text { os alunos medianos } \\
\text { como a norma. } \\
\text { Os alunos praticam } \\
\text { a solidariedade } \\
\text { mútua. } \\
\text { Os estudantes } \\
\text { tentam se comportar } \\
\text { modestamente. }\end{array}$ & $\begin{array}{l}\text { Em sociedades mais } \\
\text { masculinas, os } \\
\text { professores elogiam } \\
\text { abertamente os bons } \\
\text { alunos. } \\
\text { Os professores usam } \\
\text { os melhores alunos } \\
\text { como a norma. } \\
\text { Os alunos competem } \\
\text { entre si nas classes. } \\
\text { Os alunos tentam se } \\
\text { fazer visíveis. }\end{array}$ \\
\hline UAI & $\begin{array}{l}\text { Os alunos se sentem } \\
\text { confortáveis em } \\
\text { situações de } \\
\text { aprendizagem não- } \\
\text { estruturadas (e.g } \\
\text { objetivos vagos, } \\
\text { atribuições amplas e } \\
\text { sem tempo de } \\
\text { execução). } \\
\text { Os professores } \\
\text { podem dizer "eu não } \\
\text { sei". } \\
\text { Alunos são } \\
\text { recompensados por } \\
\text { novas abordagens } \\
\text { para resolução de } \\
\text { problemas. } \\
\text { Professores e alunos } \\
\text { devem suprimir suas } \\
\text { emoções. } \\
\text { Professores } \\
\text { interpretam } \\
\text { divergências como } \\
\text { um exercício } \\
\text { estimulante. }\end{array}$ & $\begin{array}{l}\text { Os alunos se sentem } \\
\text { confortáveis com } \\
\text { situações de } \\
\text { aprendizagem } \\
\text { estruturadas (e.g } \\
\text { objetivos precisos, } \\
\text { atribuições } \\
\text { detalhadas e com } \\
\text { tempo de execução). } \\
\text { Espera-se que os } \\
\text { professores tenham } \\
\text { todas as respostas. } \\
\text { Alunos são } \\
\text { recompensados pela } \\
\text { acurácia em resolver } \\
\text { problemas. } \\
\text { Professores e alunos } \\
\text { podem mostrar suas } \\
\text { emoções. } \\
\text { Professores } \\
\text { interpretam } \\
\text { discordância como } \\
\text { uma deslealdade } \\
\text { pessoal. }\end{array}$ \\
\hline
\end{tabular}

Os estudos de Vatrapu e Suthers ainda indicam os estilos de comunicação presentes nas diversas culturas que podem ser interessantes dentro de uma situação de aprendizagem on-line. Mais detalhes em Vatrapu e Suthers (2007).

\section{Trabalhos Relacionados}

Nesta seção são sumarizados alguns trabalhos envolvendo a pesquisa sobre os aspectos culturais relacionados principalmente as áreas de web semântica, ontologias e educação.

Chandramouli et al. (2008) apresentou a noção da Ontologia CAE-L para a modelagem do estereótipo de artefatos culturais na educação adaptativa e usou o questionário de Artefatos Culturais em Educação (do acrônimo Cultural Artifacts in Education - CAE) para coletar as informações necessárias para determinar se existe um viés cultural significativo na educação online.

Blanchard e Mizoguchi (2008) introduziram uma ontologia de topo sobre a 
cultura. Eles têm como objetivo identificar os principais constituintes a serem considerados quando se lida com qualquer tipo de questão cultural sem a necessidade de se endossar alguma estrutura de representação de uma cultura em particular. Eles trabalham no meta-nível da cultura, e usam essa abordagem para lidar com muitos sistemas tutores cientes da cultura, fornecendo formalismo para a representação cultural.

Motz et al. (2005) apresentam uma arquitetura no projeto de e-learning chamado Educa, baseado em um forte uso de ontologias para a recuperação, gestão e agrupamento de recursos eletrônicos educacionais de acordo com os aspectos culturais do usuário (veja a ontologia na figura 1), e.g. atitude, linguagem, estilos de aprendizagem e atividades. Esses aspectos culturais são especificados em uma ontologia, que segue o padrão LOM (Learning Object Metadata) e utiliza a linguagem OWL (Web Ontology Language).

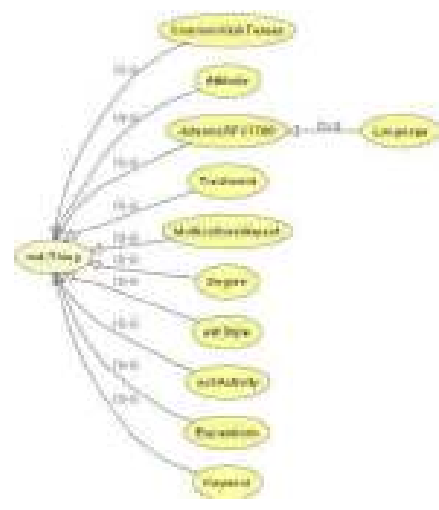

Figura 1 - ontologia do projeto EduCa

Reinecke et al. (2007) apresentam uma ontologia do modelo cultural do usuário (do acrônimo Cultural User Model Ontology CUMO) que contém informações, tais como diferentes lugares de residência, nacionalidade dos pais, idiomas, e as dimensões nacionais de Hofstede.

Reinecke et al. (2010) detalham uma ferramenta culturalmente adaptativa para criação de atividades on-line, chamada MOCCA (MOdelling Culture for Cultural Adaptivity) que é uma ferramenta disponível na web que permite aos usuários gerenciarem suas tarefas on-line. O objetivo da ferramenta é se adaptar automaticamente as preferências culturais de seus usuários - a ferramenta MOCCA não oferece conteúdo em si, mas depende de conteúdo gerado pelo usuário, e fornece a adaptação do nível de apresentação.

Os autores apresentaram dez diferentes aspectos da interação do usuário e eles observaram a influência da cultura na percepção da interface do usuário, e, posteriormente, compilaram uma lista de diretrizes de adaptação geral, e as avaliaram em um estudo e experimento com 30 participantes. $\mathrm{Na}$ figura 2 são apresentadas duas telas adaptadas da ferramenta MOCCA. À esquerda é apresentada a ferramenta MOCCA com navegação plana (com pouca profundidade) e atividades com cores distintas; à direita a ferramenta MOCCA é apresentada com uma árvore de navegação e tarefas representadas por ícones, e o sistema de ajuda demarcado.
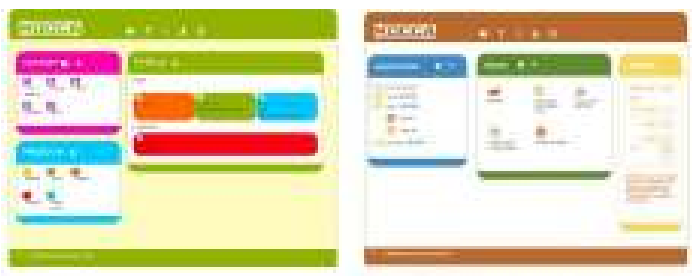

Figura 2 - Diferentes adaptações da ferramenta MOCCA (Reinecke e Bernstein 2011)

A pesquisa nos sistemas hipermídia adaptativos educacionais tem demonstrado que a consideração do contexto do usuário leva a uma melhor compreensão e personalização. Contexto é vital para melhorar a personalização nos sistemas elearning. Em um sentido mais amplo, o contexto descreve as circunstâncias pelas quais algo acontece como também a interrelação entre essas circunstâncias. Tais inter-relações fornecem uma perspectiva semântica que restringe e estreita conceitos. O processo de aprendizagem deve prover conteúdo contextualizado que deve ser ligado com o contexto da informação, podendo assim ser reutilizado em outro contexto.

Trabalhos recentes têm como objetivo de identificar os conteúdos corretos, os serviços 
certos, no lugar e no momento corretos, e na forma correta com base na situação atual do aluno, conforme apresentado em trabalhos como Barbosa et al. (2006), Yang et al. (2006), MOBIlearn (2003) e Bouzeghoub e Do Ngoc (2008). As proposições interessantes do GlobalEdu (Barbosa et al., 2006) em termos de arquitetura, por exemplo, apresenta alternativas com diferentes modelos (do aluno, do contexto e do ambiente).

A nossa pesquisa tem um ponto de vista diferente destes trabalhos, pois acreditamos que o processo de aprendizagem deve levar em consideração o contexto do usuário, e tal contexto pode descrever as perspectivas pedagógicas (estratégias de aprendizagem e objetivos educacionais), pessoais (características individuais dos usuários), tecnológicas (características tecnológicas do ambiente de aprendizagem) e culturais dos alunos (características e background), e desta forma, nós integramos os aspectos culturais, tecnológicos, educacionais e pessoais como parte de um modelo mais rico de contexto do usuário que faz sentido em uma situação especial, em um determinado momento.

Uma melhoria na informação contextual do usuário leva a uma melhor compreensão do comportamento dos usuários, a fim de enriquecer a adaptação do conteúdo, da interface, da navegação e a assistência oferecida aos usuários/alunos.

\section{Ambiente AdaptWeb ${ }^{\circledR}$}

AdaptWeb $^{\circledR} \quad$ (Ambiente de EnsinoAprendizagem Adaptativo na Web) é um sistema e-learning adaptativo que tem como objetivo adaptar o conteúdo, a apresentação e a navegação de cursos na web, de acordo com o modelo do usuário/aluno. O ambiente AdaptWeb $^{\circledR}$ é um ambiente open source, disponível no SourceForge (http://adaptweb.sourceforge.net/) e utilizado em diversas universidades (e.g. http://ead.joinville.udesc.br/adaptweb/).

O conteúdo educacional do ambiente AdaptWeb $^{\circledR}$ é modelado através de uma estrutura hierárquica de conceitos, onde os critérios de pré-requisitos são estabelecidos. Esta estrutura é definida durante a fase de autoria e depois armazenada no formato XML (Extensible Markup Language).

Os documentos XML passam por um processo de filtragem que acontece dinamicamente na interação do estudante com o ambiente, e satisfaz os critérios de adaptação representados no modelo de cada estudante em particular, baseado nas informações contidas no modelo: conhecimento, formação e preferências navegacionais e de interação (Gasparini et al., 2010). A figura 3 apresenta a tela do ambiente sem autenticação e a figura 4 mostra a tela do ambiente do aluno.

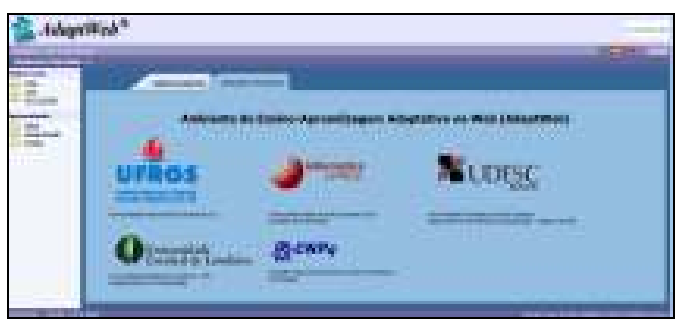

Figura 3 - Ambiente AdaptWeb ${ }^{\circledR}$

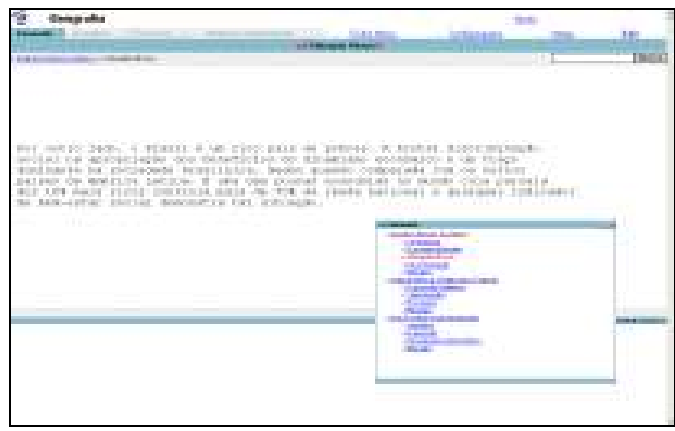

Figura 4 - Ambiente AdaptWeb ${ }^{\circledR}$ - visão do aluno no modo de navegação livre

O ambiente possui dois modos de navegação por uma disciplina: tutorial e livre. O modo de navegação tutorial respeita os pré-requisitos definidos na fase de autoria, e os alunos somente podem ir para conceitos onde seus pré-requisitos já foram adquiridos. Neste modo de navegação o aluno possui diversos instrumentos navegacionais para ser auxiliado e guiado pelo sistema.

Já o modo livre deixa habilitado todos os conteúdos de uma disciplina, e o aluno pode explorar a hierarquia da melhor maneira que o convém. Neste modo de navegação, o 
ambiente proporciona ferramentas de busca para auxiliar o aluno em sua orientação.

Atualmente uma ferramenta de diagnóstico do estilo cognitivo de aprendizagem (ECA) do aluno foi implementada e implantada no ambiente. Com esta ferramenta o professor pode descobrir o ECA segundo o modelo Felder (Felder e Brent, 2005), na dimensão ativo versus reflexivo. A tabela 2 apresenta resumidamente as dimensões do ECA do Modelo de Felder (Felder e Brent (2005).

Tabela 2 - Estilos de Aprendizagem segundo o Modelo de Felder (Felder e Brent 2005)

\begin{tabular}{|c|c|c|}
\hline Dimensão & Estilo & Descrição \\
\hline \multirow{2}{*}{ 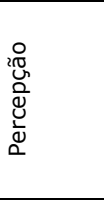 } & Sensorial & $\begin{array}{l}\text { Preferem lidar com situações } \\
\text { concretas, dados e } \\
\text { experimentos }\end{array}$ \\
\hline & Intuitivo & $\begin{array}{l}\text { Intuitivos são inovadores, } \\
\text { gostam de lidar com } \\
\text { conceitos, teorias e } \\
\text { abstrações }\end{array}$ \\
\hline \multirow{2}{*}{ 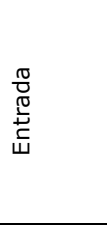 } & Visual & $\begin{array}{l}\text { Aprendem mais facilmente } \\
\text { através de figuras, } \\
\text { diagramas, fluxogramas, } \\
\text { filmes e demonstrações }\end{array}$ \\
\hline & Verbal & $\begin{array}{l}\text { Compreendem melhor as } \\
\text { informações que são } \\
\text { transmitidas por meio das } \\
\text { palavras }\end{array}$ \\
\hline \multirow{2}{*}{ 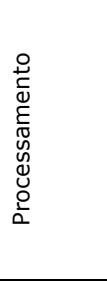 } & Ativo & $\begin{array}{l}\text { Aprendem através da } \\
\text { experimentação ativa, } \\
\text { compreendem as informações } \\
\text { mais eficientemente } \\
\text { discutindo e aplicando os } \\
\text { conceitos }\end{array}$ \\
\hline & Reflexivo & $\begin{array}{l}\text { Precisam de um tempo } \\
\text { sozinhos para pensar e } \\
\text { refletir sobre as informações } \\
\text { obtidas }\end{array}$ \\
\hline \multirow{2}{*}{ 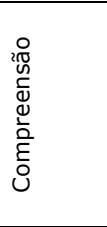 } & Seqüencial & $\begin{array}{l}\text { Aprendem melhor quando o } \\
\text { conceito é expresso de forma } \\
\text { contínua de dificuldade e } \\
\text { complexidade }\end{array}$ \\
\hline & Global & $\begin{array}{l}\text { São multidisciplinares, } \\
\text { aprendem em grandes saltos, } \\
\text { lidando de forma aleatória } \\
\text { com os conteúdos }\end{array}$ \\
\hline
\end{tabular}

Para a descoberta do ECA, são analisadas as ferramentas de comunicação Fórum de Discussão e Mural de Recados. A ferramenta analisa a interação do aluno com essas ferramentas de comunicação em cada disciplina para realizar o diagnóstico. Os resultados possíveis são: ativo, reflexivo, neutro, ou pouca interação, este significa que os dados não são suficientes para diagnosticar o ECA do aluno naquele momento. Mais detalhes em Hoehstein et al. (2011). A figura 5 apresenta a ferramenta de visualização do ECA do aluno em uma disciplina.

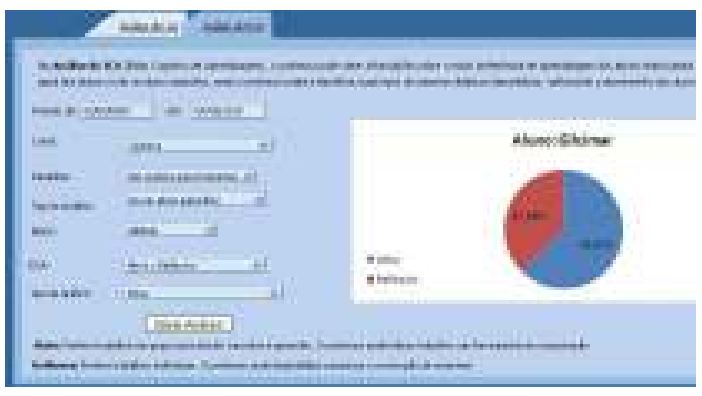

Figura 5 - Visualização do ECA do aluno em uma disciplina (Hoehstein et al. 2011)

\section{Modelo do Usuário considerando o contexto cultural}

Nosso objetivo de pesquisa tem sido investigar abordagens para modelar o perfil dos usuários e o conhecimento sobre seu contexto, e coloca a modelagem contextual em prática através de uma extensão do AdaptWeb $^{\circledR}$.

Desta forma, a arquitetura do ambiente AdaptWeb $^{\circledR}$ foi estendida para incorporar a noção de contexto, e foram desenvolvidos três serviços que são responsáveis por: (i) modelar as situações vivenciadas pelos estudantes, (ii) gerenciar e armazenar seus perfis e contextos e (iii) gerenciar seus objetos de aprendizagem correspondentes.

Esses serviços se comunicam com os mecanismos de adaptação e o serviço responsável pela modelagem do aluno emprega a nossa abordagem para modelar contexto e cultura apresentada a seguir. Mais detalhes sobre a arquitetura estendida e os serviços correlacionados em Pernas et al. (2010). A figura 6 apresenta a arquitetura estendida do ambiente AdaptWeb $^{\circledR}$. 


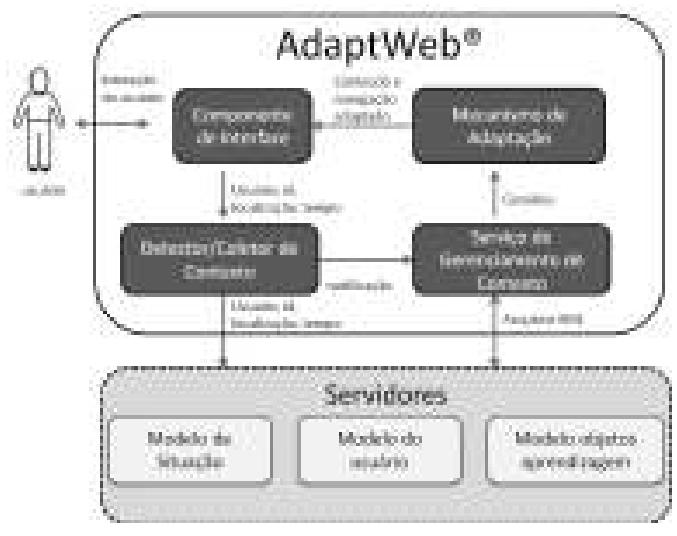

Figura 6 - Arquitetura estendida do Ambiente AdaptWeb ${ }^{\circledR}$

Nesta seção apresentamos a nossa abordagem para modelagem do contexto cultural do usuário nos sistemas e-learning. Particularmente, nós melhoramos o modelo do usuário utilizado no ambiente AdaptWeb ${ }^{\circledR}$, a fim de incorporar a noção de contexto, e por isso adotamos um modelo em três camadas (Eyharabide et al., 2009).

Neste modelo, um aluno pode estar envolvido em vários contextos que se sobrepõem, e, conseqüentemente, a atividade educativa pode ser influenciada pelas interações entre estes contextos. Nosso modelo tem três níveis de abstração: meta-modelo, modelo (ontologias), e objeto.

O nível meta-modelo é representado por uma ontologia de topo (também chamada de nível superior), descrevendo conceitos abstratos como usuário, aplicativo, situação ou data; o nível do modelo expressa as diferentes dimensões contextuais, com diferentes ontologias para descrever os elementos que compõem o contexto e, no nível mais baixo - o modelo de objeto, onde são encontradas as instâncias das ontologias de contexto, por exemplo, um nome concreto de um usuário específico (João Silva), uma disciplina específica (Interação Humano Computador), ou um determinado dispositivo (dispositivo móvel). Em outras palavras, os conceitos da ontologia de um nível são as instâncias de seu nível imediatamente superior (Eyharabide et al., 2009). A figura 7 apresenta um exemplo da abordagem proposta.
Nós personalizamos um sistema elearning para cada usuário com base nas informações disponíveis em seu modelo. As características típicas dos alunos são estendidas para incluir os contextos (i) pessoal, (ii) tecnológico, (iii) educacional e (iv) cultural; e cada dimensão é representada por uma ontologia específica.

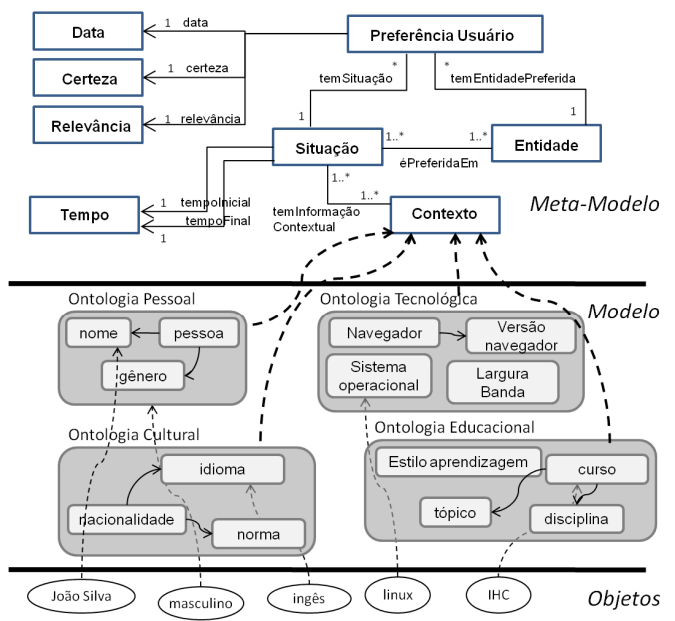

Figura 7 - Exemplo da abordagem proposta (Eyharabide et al., 2009)

A ontologia Pessoal representa as informações pessoais e de preferência de cada aluno, e as características do usuário em nosso modelo incluem: idade, escolaridade, gênero, experiências, preferências navegacionais e de assistência, e algumas informações sobre o domínio, incluindo curso de aluno (por exemplo, Ciência da Computação, Matemática, etc.), e disciplina do estudante (por exemplo Inteligência Artificial, Sistema de Banco de Dados, Engenharia de Software, etc.).

A ontologia educacional considera o conhecimento do aluno sobre o assunto a ser ensinado e os estilos de aprendizagem do Modelo de Felder (Felder e Brent, 2005).

A ontologia tecnológica está relacionada com as diferentes restrições tecnológicas e estamos preocupados especialmente com o tipo de navegador e versão, e com a conexão de rede (largura de banda), porque nos adaptamos objetos de aprendizagem em função das mesmas (e.g. apresentar ou não vídeos com alta resolução para uma conexão lenta ou discada). 
A ontologia cultural (figura 8) utiliza a nacionalidade do aluno, bem como as cinco dimensões de Hofstede (PDI, IDV, MAS, UAI, LTO), a língua materna do aluno, o grau de habilidade em outros idiomas, as experiências educacionais do aluno, detalhando se o aluno em questão realizou parte dos estudos em outro país, destacando assim a possibilidade dele ser culturalmente ambíguo (Reinecke e Bernstein, 2008), bem como a sua localização no momento (endereço IP e país), visto que um estudante pode estar em um país diferente, e então, o ambiente pode mostrar, além de objetos de aprendizagem relacionadas à sua cultura, alguns objetos da cultura em que o usuário está envolvido naquele momento.

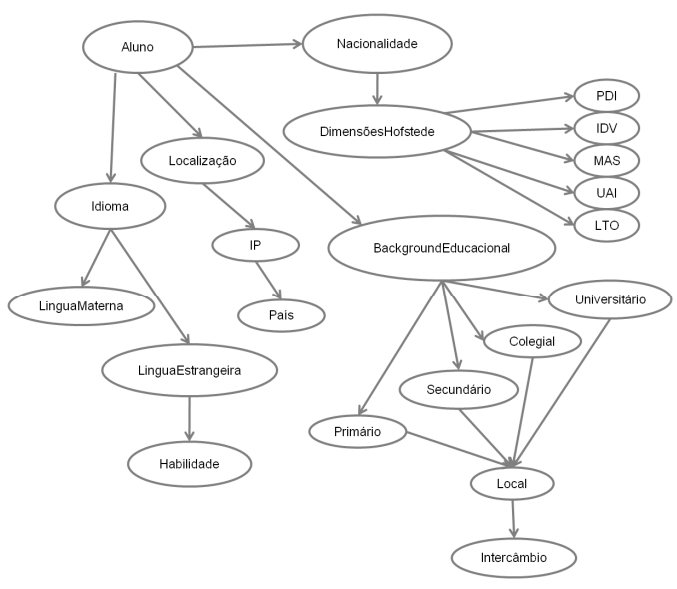

Figura 8 - Ontologia cultural

\section{Cenários de adaptação}

Nesta seção são detalhados dois cenários de aplicação para a visualização da capacidade de melhorias de adaptação e personalização que estão sendo incluídas no ambiente AdaptWeb ${ }^{\circledR}$ através da modelagem do contexto do usuário. Descrevemos aqui duas situações de aprendizagem diferentes para explicar as adaptações contextuais desenvolvidas no ambiente, e depois, destacamos como essas situações desencadeiam a correspondente adaptação contextual.

São apresentados alguns exemplos possíveis de contextos em uma disciplina chamada "Introdução à Interação HomemComputador (IHC)" para dois cursos distintos: Ciência da Computação e Engenharia. Descrevemos alguns exemplos em que o professor forneceu um conjunto de recursos (objetos de aprendizagem) e links com conteúdo diversificado sobre IHC, por exemplo: Fundamentos de IHC, Fatores Humanos, técnicas de avaliação, etc.

Para simplificar, colocamos aqui algumas variáveis do modelo do aluno: disciplina, curso, conhecimento, assunto, estilo de aprendizagem, conexão de rede, estilo de aprendizagem, nível do idioma, língua materna e país.

No contexto 1 é apresentado João, estudante de Ciência da Computação, que vive no Brasil e sua língua materna é Português, e tem um baixo nível de conhecimento na língua Inglesa. Ele está tentando aprender sobre o tópico Técnicas de Avaliação, que é explicado em Inglês. Ele está realizando exercícios sobre o conteúdo, mas infelizmente, não está obtendo resultados satisfatórios. Adicionalmente, ele possui uma conexão de rede de alta velocidade e de acordo com o Modelo de Felder, ele possui o estilo de aprendizagem ativo. O ambiente detecta através da agenda do professor que um bate-papo com os alunos foi previamente agendado para acontecer em 15 minutos. Desta forma, o sistema envia uma mensagem para o aluno, notificando-o dessa possibilidade para resolver suas dúvidas e destaca o link de "chat" na interface.

Em outro cenário, o contexto 2, é apresentada a Marie, uma estudante francesa de mestrado em Engenharia, com grande habilidade em três idiomas estrangeiros (inglês, português e espanhol).

Ela também está aprendendo sobre o assunto técnicas de avaliação, e não tem bons resultados. Ela está em uma conexão de rede de baixa velocidade e seu estilo de aprendizagem segundo Felder é reflexivo.

Em conseqüência, o AdaptWeb $^{\circledR}$ envia uma mensagem por email para o seu professor aconselhando a entrar em contato com o aluno e também modifica a ordem dos links no ambiente do aluno, colocando links relacionados a material do tipo vídeo, com resolução de baixa qualidade no final da lista, e desativando links de objetos de aprendizagem do tipo vídeo com alta 
resolução (aqueles que são muito pesados e difíceis de visualização).

Além disso, o AdaptWeb ${ }^{\circledR}$ detecta alguns links de materiais importantes sobre o assunto escritos em Inglês e Espanhol e os apresenta no topo da lista.

Em resumo, os mecanismos de adaptação do AdaptWeb ${ }^{\circledR}$ podem ser, por exemplo as seguintes ações e recomendações:

Contexto $1 \rightarrow$ "enviar notificação para o aluno apenas em Português" + "apresentar links com destaque" + "recomendar objetos de aprendizagem e conteúdos sobre o mesmo tópico (mesmo conceito na ontologia de domínio) em Português com baixo nível de dificuldade". Um exemplo possível desta adaptação é apresentado na figura 9, onde um conjunto de objetos de aprendizagem é especialmente adaptado para o aluno.

Contexto $2 \rightarrow$ "classificação e ordenação dos links" + "ocultar e desabilitar links" + "mostrar links destacados" + "apresentar e recomendar objetos de aprendizagem e conteúdos sobre o mesmo assunto escritos em Francês, Inglês, Espanhol ou Português". Um exemplo dessa adaptação é apresentado na figura 10 .

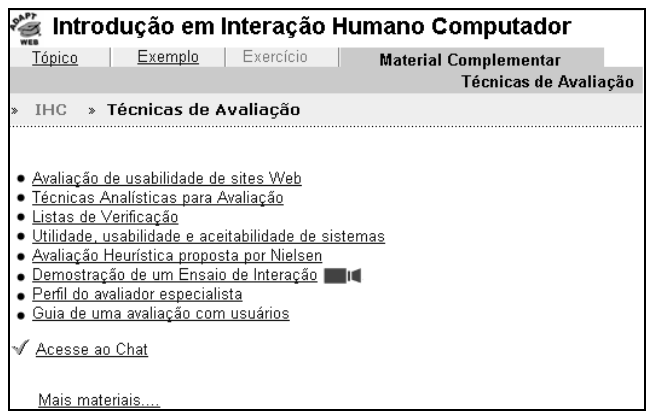

Figura 9 - AdaptWeb ${ }^{\circledR}$ adaptado ao João

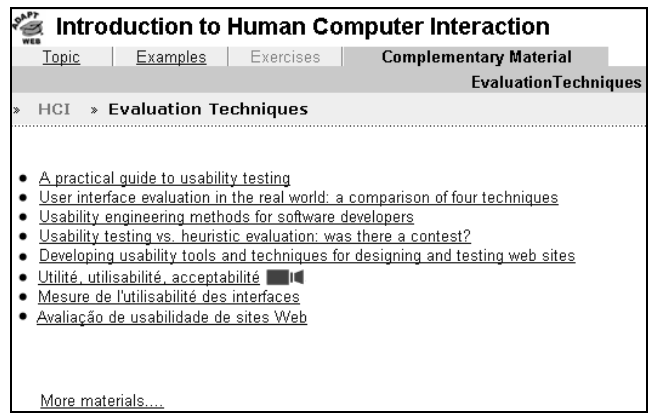

Figura 10 - AdaptWeb ${ }^{\circledR}$ adaptado a Marie

\section{Conclusão}

O aspecto cultural e a consciência da cultura em ambientes interativos são conceitos que vem ganhando evidência nas pesquisas relacionadas com diversos campos da computação.

Nos últimos anos, estamos preocupados em proporcionar suporte ao contexto cultural para sistemas de e-learning, além de outras informações contextuais do usuário. De fato, diversos pesquisadores reconhecem a importância de integrar informações de contexto nos sistemas de elearning.

Como sistemas de e-learning evoluem, eles se tornam mais sofisticados e há também um aumento na necessidade de enfrentar os desafios específicos envolvidos em incluir o conceito de cultura na bem conhecida e renomada modelagem do usuário, que ainda é adotada de forma tradicional nos ambientes adaptativos. De fato, no AdaptWeb ${ }^{\circledR}$, a modelagem do contexto amplia as técnicas de modelagem tradicionais do usuário, lidando explicitamente com aspectos que podem ter uma influência significativa no processo de aprendizagem suportado por um sistema elearning, como os aspectos pessoais, educacionais, tecnológicos e notavelmente cultural.

É nossa suposição que a modelagem do contexto do usuário leva ao design do sistema que oferece conteúdos de aprendizagem mais apropriados e serviços que satisfazem as necessidades dos alunos, automaticamente adaptando seu comportamento para tais mudanças. A maioria dos trabalhos e pesquisas sobre sensibilidade ao contexto está associada com as questões de contexto tecnológico como a localização ou plataforma. Em nosso trabalho, as características típicas de modelagem do aluno são ampliadas para incluir as dimensões de contexto pessoal, educacional, tecnológico e cultural. Cada dimensão contextual é representada por uma ontologia diferente. Cenários são utilizados para representar situações específicas e estamos especialmente interessados em modelar cenários, porque eles mudam de acordo com o contexto. 
Propomos o uso de ontologias para modelar estas informações contextuais, como um modelo de três camadas para captar os diferentes níveis de detalhe. Este artigo descreve 0 uso das ontologias para representar explicitamente o contexto como uma extensão da modelagem tradicional do aluno. Nosso objetivo é incrementar ainda mais a real capacidade dos sistemas de personalização de fazer uso de ontologias para modelar contexto do aluno em diferentes cenários, adaptando o conteúdo, a navegação e apresentação dos sistemas elearning.

Uma vez que a sensibilidade e ciência do contexto cultural em sistemas e-learning não é ainda um conceito amadurecido, uma contribuição geral deste trabalho é um novo ponto de vista a respeito de como deve ser uma infra-estrutura tecnológica que visa dar apoio para a consciência cultural em sistemas de e-learning sensíveis ao contexto do aluno.

Como contribuição prática, as ontologias e algumas melhorias das capacidades da personalização no ambiente AdaptWeb ${ }^{\circledR}$ foram projetadas e implementadas a fim de avaliar mais sistematicamente a validade de alguns pressupostos e proposições.

Nós não temos a pretensão de que os aspectos de contexto culturais aqui descritos neste trabalho representam um tratamento completo da cultura nos sistemas elearning, mas esperamos que este trabalho proporcione um impulso nessa direção.

Nosso modelo foi incluído no ambiente AdaptWeb $^{\circledR}$ para permitir uma experimentação real. Uma característica obtida na modelagem do contexto do usuário foi a representação do contexto tão independente quanto possível do conteúdo educacional, para que este possa ser reutilizado e adaptado às mudanças de contexto, permitindo aos sistemas elearning dinamicamente compor, reutilizar e adaptar objetos de aprendizagem. Atualmente, este modelo está sendo avaliado com alunos reais em cursos presenciais do ambiente AdaptWeb ${ }^{\circledR}$.

\section{Agradecimentos}

Este trabalho foi parcialmente financiado pelo CNPq (Conselho Nacional de Desenvolvimento Científico e Tecnológico) (CT-Info/CNPq 17/2007) e pelos projetos Adaptar-SUR 022/07 (CAPES, Brasil), AdContext 547-07 (CAPES-COFECUB), PROSUL CNPq 08/2010 "Avaliação multicultural do comportamento em ambiente Web" e projeto Universal MCT/CNPq 14/2010 "Adaptabilidade da interação na Web".

\section{Referências}

ABOU-ZEID, El-Sayed. A culturally aware model of inter-organizational knowledge transfer. Knowledge Management Research \& Practice, 3, pp. 146-155, 2005.

BARBOSA, D. N. F.; AUGUSTIN, I.; BARBOSA, J. L. V.; YAMIM, A. C.; DA SILVA, L. C.; Fernando, C.; GEYER, R. Learning in a Large-Scale Pervasive Environment. 4th IEEE Conference on Pervasive Computing and Communications Workshops, (PERCOM), pp. 226-230, 2006.

BLANCHARD, E. G.; MIZOGUCHI, R. Designing Culturally-Aware Tutoring Systems: Towards an Upper Ontology of Culture. Workshop on Culturally-Aware Tutoring Systems (CATS), ITS 2008, pp 23-34, 2008.

BOUZEGHOUB, A., DO NGOC, K. A situation based meta-data for describing pervasive learning objects. 1st International Conference on Mobile Learning, University of Wolverhampton, Ironbridge, UK, 2008.

BRUSILOVSKY, P.; MILLÁN, E. User Models for Adaptive Hypermedia and Adaptive Educational Systems The Adaptive Web, LNCS 4321, pp. 3-53, Springer-Verlag, 2007.

BRUSILOVSKY, P., PEYLO, C. Adaptive and intelligent web-based educational systems. International Journal of Artificial Intelligence in Education, 13 (2-4): 159 - 172, 2003. 
CHANDRAMOULI, K.; STEWART, C.; BRAILSFORD, T.; IZQUIERDO, E. CAE-L: An Ontology Modelling Cultural Behaviour in Adaptive Education. 3th International Workshop on Semantic Media Adaptation and Personalization, IEEE ,183-188, 2008.

CLEMMENSEN, T.; HERTZUM, M.; HORNBAEK, K.; SHI, Q.; YAMMIYAVAR, P. Cultural Cognition in Usability Evaluation. Interacting with Computers, 21(3), pp. 212-220, 2009.

DORMANN, C.; CHISALITA, C. Cultural Values in Web Site Design. European Conference on Cognitive Ergonomics, 2002.

EYHARABIDE. V.; GASPARINI, I.; SCHIAFFINO, S.; PIMENTA, M. S.; AMANDI, A. Personalized e-Learning Environments: Considering Students' Contexts. 9th World Conference on Computers in Education (WCCE), IFIP AICT, v. 302, 48-57, 2009.

FELDER, R.; BRENT, R. Understanding Student Differences. Journal of Engineering Education, 94 (1), 5772, 2005.

GASPARINI, I; PIMENTA, M. S.; PALAZZO M. DE OLIVEIRA, J. Vive La différence!: a survey of culturalaware issues in HCI. X Simpósio Brasileiro de Fatores Humanos em Sistemas Computacionais (IHC) e V Latin American Conference on Human Computer Interaction (CLIHC), 2011, Porto de Galinhas, Pernambuco.

GASPARINI, I; PIMENTA, M. S.; PALAZZO M. DE OLIVEIRA, J.; KEMCZINSKI, A. Usability in na Adaptive elearning environment: Lessons from AdaptWeb. IEEE Learning Technology Newsletter, v. 2, p. 13-16, 2010.

HEIMGÄRTNER, R. Towards cultural adaptability in driver information and -assistance systems. Usability and Internationalization. Global and Local User Interfaces, 4560:372-381, 2007.

HOEHSTEIN, G.; KEMCZINSKI, A.; GASPARINI, I.; PIMENTA, M. S. Diagnóstico do Estilo de Aprendizagem do Aluno a partir de Ferramentas de Comunicação, VI Congresso Ibero-americano de Telemática (CITA), Cadernos de Informática, v. 6, n.1, 2011, pp. $95-100$

HOFSTEDE, Geert. Cultures and organizations: software of the mind. 2nd ed. New York: McGraw-Hill, 2005.

HOFSTEDE, Geert. Culture's Consequences: comparing values, behaviors, institutions, and organizations across nations. 2nd ed. Thousand Oaks, CA: SAGE Publications, 2001.

MOBIlearn, 2003. MOBIlearn final report, viewed January 2007, http://www.mobilearn.org

MOTZ, R. GUZMÁN, J.; DECO, C.; BENDER, C. Applying Ontologies to Educational Resources Retrieval driven by Cultural Aspects. Journal of Computer Science \& Technology (JCS\&T), 5(4), pp 279-284, 2005.

PERNAS, A. M.; GASPARINI, I.; BOUZEGHOUB, A.; PIMENTA, M. S.; WIVES, L. K.; PALAZZO M. DE OLIVEIRA, J. From an e-learning to an u-learning environment. $2^{\text {nd }}$ International Conference on Computer Supported Education (CSEDU), 2010, pp. 180- 185.

REINECKE, K.; BERNSTEIN, A. Improving Performance, Perceived Usability, and Aesthetics with Culturally Adaptive User Interfaces. Transactions on Computer-Human Interaction, 2011.

REINECKE, K. SCHENKEL, S.; BERNSTEIN, A. Modeling a User's Culture. The Handbook of Research in Culturally-Aware Information Technology: Perspectives and Models, IGI Global, 2010.

REINECKE, K.; BERNSTEIN, A. Predicting user interface preferences of culturally ambiguous users. Proceeding of $26^{\text {th }}$ Conference Extended Abstracts on Human Factors in Computing Systems - CHI, 2008.

REINECKE, K.; REIF, G; BERNSTEIN, A. Cultural User Modeling With CUMO: An Approach to Overcome the Personalization Bootstrapping Problem. Workshop on Cultural Heritage on the Semantic Web, ISWC, 2007.

REINECKE, K ; BERNSTEIN, A. Culturally Adaptive Software: Moving Beyond Internationalization. HCI International (HCII), Springer, 2007. 
VATRAPU, R.; SUTHERS, D. Culture and Computers: A Review of the Concept of Culture and Implications for Intercultural Collaborative Online Learning. In: T. Ishida, S.R. Fussell, and P.T.J.M. Vossen (Eds.): IWIC 2007, LNCS 4568, pp. 260-275, Springer-Verlag Berlin Heidelberg 2007.

YANG, S.J.H.; HUANG, A.P.M.; CHEN, Rick; TSENG, Shian-Shyong; SHEN, Yen-Shih. Context Model and Context Acquisition for Ubiquitous Content Access in ULearning Environments, IEEE International Conference on Sensor Networks, Ubiquitous, and Trustworthy Computing, 2, pp. 78-83, 2006.

ZAHARIAS, P. Cross-Cultural Differences in Perceptions of e-leaning Usability: An Empirical Investigation. International Journal of Technology and Human Interaction, 4(3), IGI Global, 2008.

Recebido em maio de 2011

Aprovado para publicação em junho de 2011

Isabela Gasparini

Departamento de Ciência da Computação - Universidade do Estado de Santa Catarina - UDESC, Brasil, isabela@joinville.udesc.br Doutoranda do Programa de Pós-Graduação em Ciência da Computação Universidade Federal do Rio Grande do Sul - UFRGS, Porto Alegre - Brasil. E-mail: igasparini@inf.ufrgs.br Avanilde Kemczinski

Departamento de Ciência da Computação - Universidade do Estado de Santa Catarina - UDESC, Florianópolis - Brasil. E-mail:avanilde@joinville.udesc.br

\section{Marcelo Soares Pimenta}

Programa de Pós-Graduação em Ciência da Computação - Universidade Federal do Rio Grande do Sul UFRGS, Porto Alegre - Brasil. E-mail: mpimenta@inf.ufrgs.br

José Palazzo Moreira de Oliveira

Programa de Pós-Graduação em Ciência da Computação - Universidade Federal do Rio Grande do Sul UFRGS, Porto Alegre - Brasil, E-mail: palazzo@inf.ufrgs.br 